\title{
ON THE PROPAGATION OF MAXIMALLY DISSIPATIVE PHASE BOUNDARIES IN SOLIDS
}

\author{
BY \\ ROHAN ABEYARATNE (Massachusetts Institute of Technology, Cambridge, Massachusetts) \\ AND
}

JAMES K. KNOWLES (California Institute of Technology, Pasadena, California)

\begin{abstract}
This paper is concerned with the kinetics of propagating phase boundaries in a bar made of a special nonlinearly elastic material. First, it is shown that there is a kinetic law of the form $f=\varphi(\dot{s})$ relating the driving traction $f$ at a phase boundary to the phase boundary velocity $\dot{s}$ that corresponds to a notion of maximum dissipation analogous to the concept of maximum plastic work. Second, it is shown that a modified version of the entropy rate admissibility criterion can be described by a kinetic relation of the above form, but with a different $\varphi$. Both kinetic relations are applied to the Riemann problem for longitudinal waves in the bar.
\end{abstract}

1. Introduction. Recently [1], we considered a one-dimensional dynamical theory of an elastic bar composed of a material which could undergo phase transitions. For the class of materials considered in [1], the stress-strain relation is such that stress at first increases with increasing strain, then decreases and finally increases again; the different branches of the stress-strain curve are identified with different phases of the material. A propagating strain discontinuity in a bar composed of such a material can be either a shock wave or a phase boundary, according to whether the particles separated by the discontinuity are of the same phase or of distinct phases. In addition to the basic field equations and jump conditions associated with momentum balance and kinematic compatibility, we imposed in [1] the entropy admissibility requirement that follows from the second law of thermodynamics at each propagating strain discontinuity. In the form employed in [1], this requirement states that the product of the velocity $\dot{s}$ of the discontinuity and an associated driving traction $f$ must be nonnegative: $f \dot{s} \geq 0$.

If, in contrast to the case treated in [1], the material of the bar is such that stress is a monotonically increasing, strictly convex or strictly concave function of strain, then phase transformations cannot occur, and all propagating strain discontinuities are shock waves. For a bar made of such a material, it follows from a result of Oleinik [2] that the Cauchy problem for the associated field equations and jump conditions

Received September 19, 1990.

(C) 1992 Brown University 
has at most one solution that fulfills the entropy admissibility requirement (see [3] for a discussion of Oleinik's theorem and related results). On the other hand, for materials such as those considered in [1] that do not satisfy these conditions, the Cauchy problem need no longer have a unique solution, even with the entropy admissibility requirement in force (see the remarks of Dafermos [4] in this connection). We view this lack of uniqueness as arising from a constitutive deficiency in the theory, reflecting the need to specify two additional pieces of constitutive information pertaining to phase boundaries: a nucleation criterion for the initiation of a phase transition and a kinetic relation that controls the rate at which the phase transition proceeds. The importance of nucleation and kinetics has long been recognized in the materials science literature concerning phase transformations [5].

The form of the kinetic relation employed in [1] and to be used here is one in which the driving traction is a materially-determined function of the velocity of the moving phase boundary: $f=\varphi(\dot{s})$. The nucleation criterion given in [1] specifies a critical level of driving traction that signals the onset of a phase transformation. We had previously explored the continuum-mechanical implications of such a kinetic relation and nucleation criterion in the context of one-dimensional quasi-static motions [6]. A more general discussion of kinetic relations for a thermoelastic material undergoing a thermomechanical process in a three-dimensional setting with inertia effects taken into account may be found in [7].

For a special material whose rising-falling-rising stress-strain curve is piecewise linear (the "trilinear material"), we showed in [1] that, for the Riemann problem, the extent of lack of uniqueness of solution remaining after imposition of the entropy admissibility requirement is precisely that needed to accommodate a nucleation criterion and a kinetic relation at phase boundaries that propagate subsonically with respect to both phases. We also showed that, for this special material, a kinetic relation cannot be prescribed at phase boundaries that move supersonically with respect to the phase with lower sound wave speed.

In the present paper, we are concerned with two special kinetic relations of the form $f=\varphi(\dot{s})$. First, we introduce the maximally dissipative kinetic relation, which is based on a notion of maximum dissipation analogous to the concept of maximum plastic work in the theory of elastic-plastic solids $[8,9]$. The second kinetic law to be discussed here is related to a special selection criterion, to be described below, for singling out solutions to dynamical problems. We study the implications of these kinetic relations for a Riemann problem for the trilinear material.

To address cases in which the stress fails to be a monotonically increasing function of strain that is also strictly convex or concave, other workers in this area have sought to replace the entropy admissibility requirement with various more discriminating conditions that would serve to select solutions to the Cauchy problem. Among such selection criteria, perhaps the two that are most often encountered in applications are the "viscosity-capillarity condition" studied by Shearer [10, 11], Slemrod [12, 13], Truskinovsky [14, 15], and others, and variants of the "entropy rate admissibility criterion" proposed by Dafermos $[16,17]$ and investigated in connection with phase transitions by Hattori [18, 19], James [20], and Pence [21]. For the trilinear elastic 
bar, the relation between a selection criterion of the viscosity-capillarity type and the kinetics of phase transitions was recently explored in [22], where it was shown that imposing this selection criterion is equivalent to the prescription of a kinetic relation $f=\varphi(\dot{s})$ with a particular choice of $\varphi$. In the present paper, we show-again for the trilinear material-that the "entropy rate shock admissibility criterion" stated by Dafermos in [17], if suitably modified, is also equivalent to a kinetic relation of the form $f=\varphi(\dot{s})$. The three kinetic response functions $\varphi$ for the maximally dissipative kinetic relation, the viscosity-capillarity condition, and the modified entropy rate shock admissibility criterion are all distinct.

It is important to emphasize that the entropy admissibility requirement $f \dot{s} \geq 0$ at strain discontinuities follows from a fundamental physical principle and is thus applicable to all materials. In contrast, selection criteria such as the viscosity-capillarity condition, the entropy rate admissibility criterion, and the maximally dissipative kinetic relation do not enjoy this universal status and thus can at best be constitutive statements that pertain to a particular material or class of materials. Moreover, to qualify as a constitutive assertion, any such criterion must not only be consistent with the entropy admissibility condition, but it must be a problem-independent, local restriction that can be stated in terms of individual particles of the continuum at hand. Kinetic relations of the form $f=\varphi(\dot{s})$ meet these requirements and represent constitutive statements that, for a given material, are applicable at all phase boundaries arising in any boundary-initial-value problem in the theory of longitudinal waves in bars. The same is true of the viscosity-capillarity condition and the modified entropy rate shock admissibility criterion.

In the next section we set out the basic field equations, jump conditions, and the entropy admissibility requirement. In Sec. 3 we describe the trilinear material and review the local properties of strain discontinuities in the simplest one-dimensional theory of elastic bars. After describing the notion of a kinetic relation at the beginning of Sec. 4, we introduce the local concept of maximum dissipation rate and deduce the corresponding maximally dissipative kinetic relation. In Sec. 5, we consider a particular Riemann problem and, for given initial data, we determine explicitly the set $\Omega$ of all solutions of this problem that satisfy the entropy admissibility condition. Each solution in this set involves a single phase boundary propagating with a constant velocity $\dot{s}$ that is not determined by the initial data; indeed, $\Omega$ comprises a oneparameter family of solutions, parameter $\dot{s}$. Sections 6 and 7 are respectively devoted to the following two questions concerning $\Omega$ : first, does imposition of the maximally dissipative kinetic relation of Sec. 4 pick out a unique value of $\dot{s}$, and hence a unique solution in $\Omega$, for the given initial data? Second, for given initial data, is there a unique value of $\dot{s}$, and hence a unique solution, that maximizes the dissipation rate $f \dot{s}$ over the solutions in $\Omega$ ? We show that, while the answer to each of these questions is affirmative, the solutions selected by the two procedures are in general different. In the present context, the selection criterion implicit in the second of these two questions is equivalent to a modified version of the entropy rate shock admissibility criterion of Dafermos [17]. 
Since the two kinetic relations to be discussed here refer to related but distinct notions of maximum dissipation rate, we are confronted with some terminological pitfalls. To help avoid these, we shall refer to the kinetic relation that mimics maximum plastic work as the maximally dissipative kinetic relation. The second kinetic law we shall speak of as associated with the modified entropy rate shock admissibility criterion. We shall consistently refer to the condition that the entropy of a particle cannot decrease upon crossing a strain discontinuity $(f \dot{s} \geq 0)$ as the entropy admissibility requirement.

2. Preliminaries. Consider an elastic bar of unit cross-sectional area occupying the interval $(-\infty, \infty)$ in an unstressed reference configuration. In a longitudinal motion of the bar, the particle located at $x$ in the reference configuration is carried to the point $x+u(x, t)$ at time $t$; the displacement $u$ is required to be continuous and to have piecewise continuous first and second derivatives on $(-\infty, \infty) \times[0, \infty)$. At points $(x, t)$ in space-time where $u_{x}$ and $u_{t}$ exist, we let

$$
\gamma=u_{x}, \quad v=u_{t}
$$

denote strain and particle velocity, respectively. In order to assure that the mapping $x \rightarrow x+u(x, t)$ be invertible at each $t$, we require that $\gamma(x, t)>-1$. The stress $\sigma(x, t)$ is related to the strain through

$$
\sigma=\hat{\sigma}(\gamma)
$$

where the stress response function $\hat{\sigma}$ is determined by the material. The mass density $\rho$ of the material in the reference configuration is taken to be constant.

At points where $\gamma$ and $v$ are smooth, balance of linear momentum and the smoothness properties of $u$ require that

$$
\begin{gathered}
\hat{\sigma}^{\prime}(\gamma) \gamma_{x}-\rho v_{t}=0, \\
v_{x}-\gamma_{t}=0 .
\end{gathered}
$$

If either $\gamma$ or $v$ is discontinuous across the curve $x=s(t)$ in the $(x, t)$-plane, the jump conditions

$$
\begin{gathered}
{[[\sigma]]=-\rho[[v]] \dot{s},} \\
{[[\gamma]] \dot{s}=-[[v]]}
\end{gathered}
$$

must be satisfied, where for any function $g(x, t)$, [[g]] $=g(s(t)+, t)-g(s(t)-, t)$. The jump condition (2.5) comes from the balance of linear momentum; (2.6) follows from the smoothness of $u(x, t)$.

The basic field equations and jump conditions (2.3)-(2.6) do not guarantee that the instantaneous dissipation rate during a motion is nonnegative. In order to assure this, one must impose an additional requirement at each discontinuity. To this end, let

$$
W(\gamma)=\int_{0}^{\gamma} \hat{\sigma}\left(\gamma^{\prime}\right) d \gamma^{\prime}, \quad \gamma>-1
$$

be the strain energy per unit reference volume for the material of the bar. Consider the restriction of the motion to the time interval $\left[t_{1}, t_{2}\right]$ and to the piece of the bar 
that occupies the interval $\left[x_{1}, x_{2}\right]$ in the reference configuration. Suppose that $\gamma$ and $v$ are smooth on $\left[x_{1}, x_{2}\right] \times\left[t_{1}, t_{2}\right]$ except at the moving discontinuity $x=s(t)$. Let $E(t)$ and $D(t)$ denote, respectively, the total mechanical energy and the rate of dissipation of mechanical energy at time $t$ associated with the piece of the bar under consideration:

$$
\begin{gathered}
E(t)=\int_{x_{1}}^{x_{2}}\left[W(\gamma(x, t))+\frac{1}{2} \rho v^{2}(x, t)\right] d x \\
D(t)=\sigma\left(x_{2}, t\right) v\left(x_{2}, t\right)-\sigma\left(x_{1}, t\right) v\left(x_{1}, t\right)-\dot{E}(t) .
\end{gathered}
$$

A direct calculation using (2.3)-(2.9) establishes the following alternative expression for the dissipation rate in terms of local quantities at the strain discontinuity:

$$
D(t)=f(t) \dot{s}(t),
$$

where $f(t)$ is given by

$$
f=\hat{f}(\bar{\gamma}, \stackrel{+}{\gamma}) \equiv \int_{\bar{\gamma}}^{+} \hat{\gamma} \hat{\sigma}(\gamma) d \gamma-\frac{1}{2}[\hat{\sigma}(\stackrel{+}{\gamma})+\hat{\sigma}(\bar{\gamma})](\dot{\gamma}-\bar{\gamma}),
$$

and $\stackrel{t}{\gamma}(t)=\gamma(s(t) \pm, t)$ are the strains on the two sides of the discontinuity. If there is no jump in strain at $x=s(t)$, then (2.11) shows that $f=0$, and so $D$ vanishes. Since (2.9) and (2.10) give

$$
\sigma\left(x_{2}, t\right) v\left(x_{2}, t\right)-\sigma\left(x_{1}, t\right) v\left(x_{1}, t\right)+(-f(t)) \dot{s}(t)=\dot{E}(t),
$$

we may view $-f(t) \dot{s}(t)$ as the rate of work done on the bar by the moving discontinuity, and thus speak of $f$ as the driving traction-i.e., the driving force per unit cross-sectional area-acting on the discontinuity. According to (2.11), the driving traction $f$ may be interpreted geometrically as the difference between the area under the stress-strain curve between the strains $\bar{\gamma}$ and $\stackrel{+}{\gamma}$ and the area of the trapezoid determined by $\bar{\gamma}, \stackrel{+}{\gamma}, \hat{\sigma}(\bar{\gamma})$, and $\hat{\sigma}(\stackrel{+}{\gamma})$.

In order to guarantee that the instantaneous dissipation rate associated with every piece of the bar is nonnegative during the motion, one must enforce the additional requirement

$$
f(t) \dot{s}(t) \geq 0
$$

at each strain discontinuity and at all times. If the material is viewed as being thermoelastic, and if we make the assumption-however unrealistic-that the motion takes place isothermally at a temperature $\theta$, then one can show that the rate of entropy production at time $t$ associated with the piece $\left[x_{1}, x_{2}\right]$ of the bar is $D(t) / \theta$. Thus, under these conditions, the restriction (2.13), with $f$ given by (2.11), is a consequence of the second law of thermodynamics; it is equivalent to the assertion that the entropy of a particle cannot decrease as the particle crosses a strain discontinuity. We refer to (2.13) as the entropy admissibility requirement. 
3. Trilinear material. Local properties of phase boundaries and shock waves. In order to allow for the possible occurrence of stationary phase boundaries in a bar, one considers a material whose stress response function $\hat{\sigma}(\gamma)$ at first increases monotonically, then decreases, and finally increases again as $\gamma$ increases from the value $\gamma=-1$. In the present study we restrict attention to the special case in which $\hat{\sigma}(\gamma)$ has the trilinear form shown in Fig. 1. Although some of the results of this paper apply only for this trilinear material, the discussion in the present section, as well as certain subsequent results, can be generalized to any rising-falling-rising stress-strain curve.

We shall say that a particle of the bar labeled by $x$ is in phase 1, 2, or 3 at time $t$ according to whether the strain $\gamma(x, t)$ lies in the interval $\left(-1, \gamma_{M}\right],\left(\gamma_{M}, \gamma_{m}\right)$, or $\left[\gamma_{m}, \infty\right)$, respectively, corresponding to the three branches of the stress-strain curve in Fig. 1. In addition, a strain discontinuity will be said to be of $p, q$-type if $\bar{\gamma}$ is a phase- $p$ strain and $\stackrel{+}{\gamma}$ is a phase- $q$ strain. If $p=q$, we call the discontinuity a shock wave, while if $p \neq q$ we refer to it as a phase boundary.

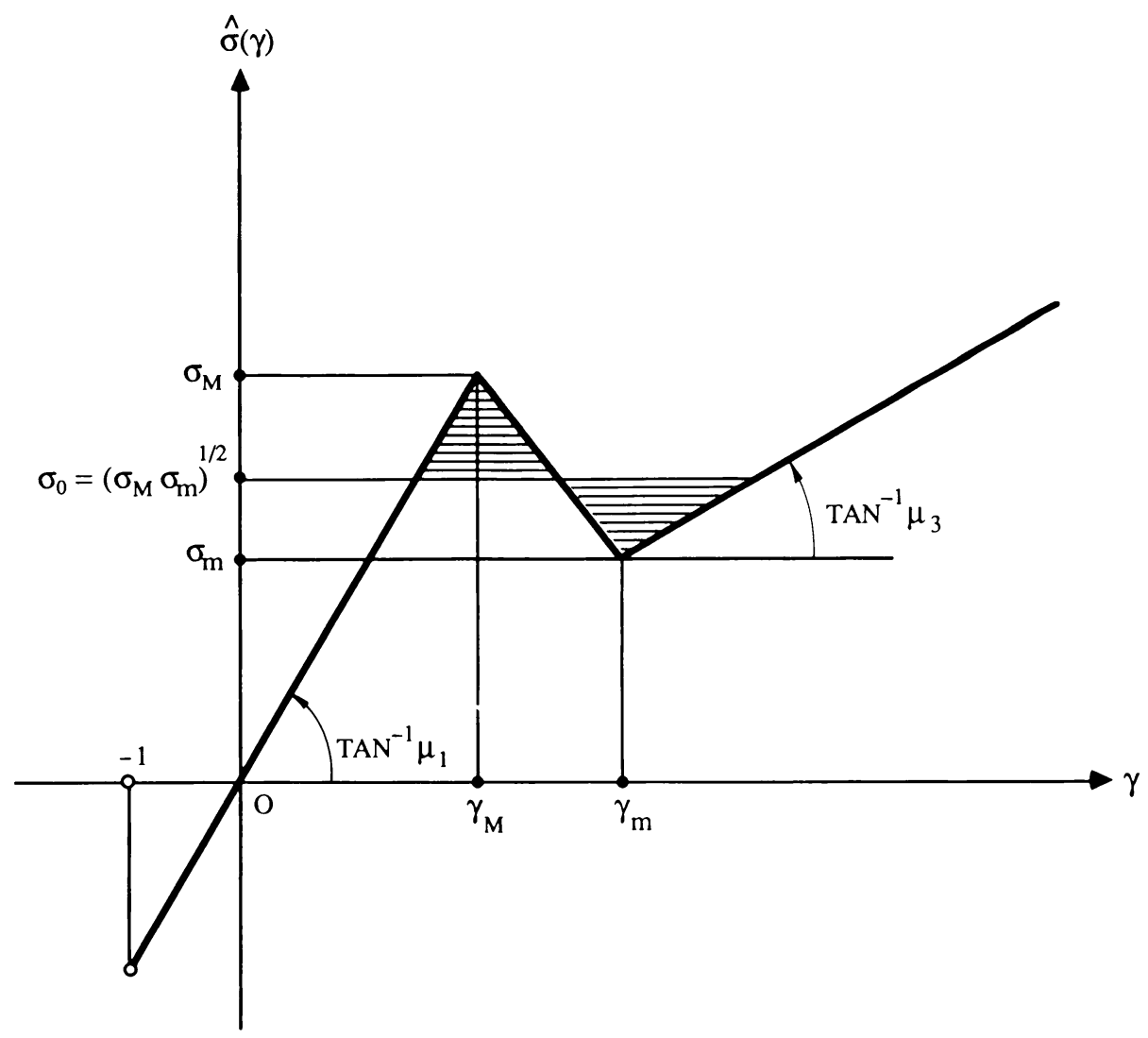

FIG. 1. Stress-strain curve for the trilinear material. 
At a moving discontinuity $x=s(t)$, the jump conditions (2.5), (2.6) imply

$$
\begin{gathered}
\rho \dot{s}^{2}=\frac{\hat{\sigma}(\stackrel{+}{\gamma})-\hat{\sigma}(\bar{\gamma})}{\dot{+}-\bar{\gamma}}, \\
{[\hat{\sigma}(\stackrel{+}{\gamma})-\hat{\sigma}(\bar{\gamma})]\left(+\frac{\hat{\gamma}}{-\gamma} \bar{\gamma}\right)=\rho\left(+\frac{v}{v}-\bar{v}\right)^{2} .}
\end{gathered}
$$

The right side of (3.1) must necessarily be nonnegative for any pair of strains $\bar{\gamma}, \stackrel{+}{\gamma}$ that can occur at a strain jump. Conversely, if $\bar{\gamma}, \stackrel{+}{\gamma}$ are numbers in $(-1, \infty)$ such that the right side of (3.1) is nonnegative, then it is possible to find numbers, $\bar{v}, \stackrel{+}{v}$, and $\dot{s}$ such that the pair of jump conditions (2.5), (2.6) are satisfied.

The requirement (3.1) rules out shock waves of 2,2-type. In addition, for the trilinear material, it shows that shock waves of 1,1-type and 3,3-type have constant propagation speeds $\pm c_{1}$ and $\pm c_{3}$, respectively, where

$$
c_{1}=\left(\mu_{1} / \rho\right)^{1 / 2}, \quad c_{3}=\left(\mu_{3} / \rho\right)^{1 / 2}<c_{1} .
$$

At a shock wave of either 1,1- or 3,3-type, the linearity of the stress response function $\hat{\sigma}(\gamma)$ between the strains $\bar{\gamma}$ and $\stackrel{+}{\gamma}$ allows one to infer from (2.11) that the driving traction $f$ vanishes. In particular, the entropy admissibility requirement $(2.13)$ is automatically satisfied at such a shock wave.

Turning next to phase boundaries, we suppose that $\bar{\gamma}$ and $\stackrel{+}{\gamma}$ belong to different phases. Since we shall not consider cases in which either $\bar{\gamma}$ or $\stackrel{+}{\gamma}$ is in phase 2 (the "unstable phase"), we may without loss of generality take $\bar{\gamma}$ to be in phase 1 and $\stackrel{+}{\gamma}$ in phase 3 , so that the associated discontinuity is a 1,3-phase boundary. When specialized to this case and to the trilinear material of Fig. 1, (3.1) becomes

$$
\dot{s}^{2}=\frac{c_{3}^{2+}-c_{1}^{2} \bar{\gamma}}{\dot{\gamma}-\bar{\gamma}} \text {. }
$$

In the $(\bar{\gamma}, \stackrel{+}{\gamma})$-plane, the set of pairs $(\bar{\gamma}, \stackrel{+}{\gamma})$ for which $\bar{\gamma}$ is in phase $1, \stackrel{+}{\gamma}$ is in phase 3, and the right side of (3.4) is nonnegative is represented by the region $\Gamma$ shown partially hatched and partly shaded in Fig. 2. At any point on the boundary segment $B C$, the numerator in (3.4) vanishes, so that $\dot{s}=0$; the corresponding phase boundary is thus instantaneously stationary. The point $M$ in the figure corresponds to the so-called Maxwell state, which is the special equilibrium phase mixture for which $\bar{\gamma}=\sigma_{0} / \mu_{1}$ and $\stackrel{+}{\gamma}=\sigma_{0} / \mu_{3}$, where $\sigma_{0}=\left(\sigma_{M} \sigma_{m}\right)^{1 / 2}$ is the stress for which the hatched areas in Fig. 1 are equal.

The driving traction $f$ acting on a phase boundary of 1,3-type can be found from (2.11) and the explicit form of $\hat{\sigma}(\gamma)$ for the trilinear material:

$$
f=\hat{f}(\bar{\gamma}, \stackrel{+}{\gamma})=\frac{1}{2}\left(\mu_{1}-\mu_{3}\right)\left(\gamma_{M} \gamma_{m}-\bar{\gamma} \dot{\gamma}\right) \text {. }
$$

It follows that the driving traction vanishes on the hyperbola $\bar{\gamma} \dot{\gamma}=\gamma_{M} \gamma_{m}$ in the $(\bar{\gamma}, \stackrel{+}{\gamma})$-plane. Thus according to (2.10), points in $\Gamma$ that lie on this hyperbola correspond to values of $\bar{\gamma}$ and $\stackrel{+}{\gamma}$ for which the associated phase boundary $x=s(t)$ 


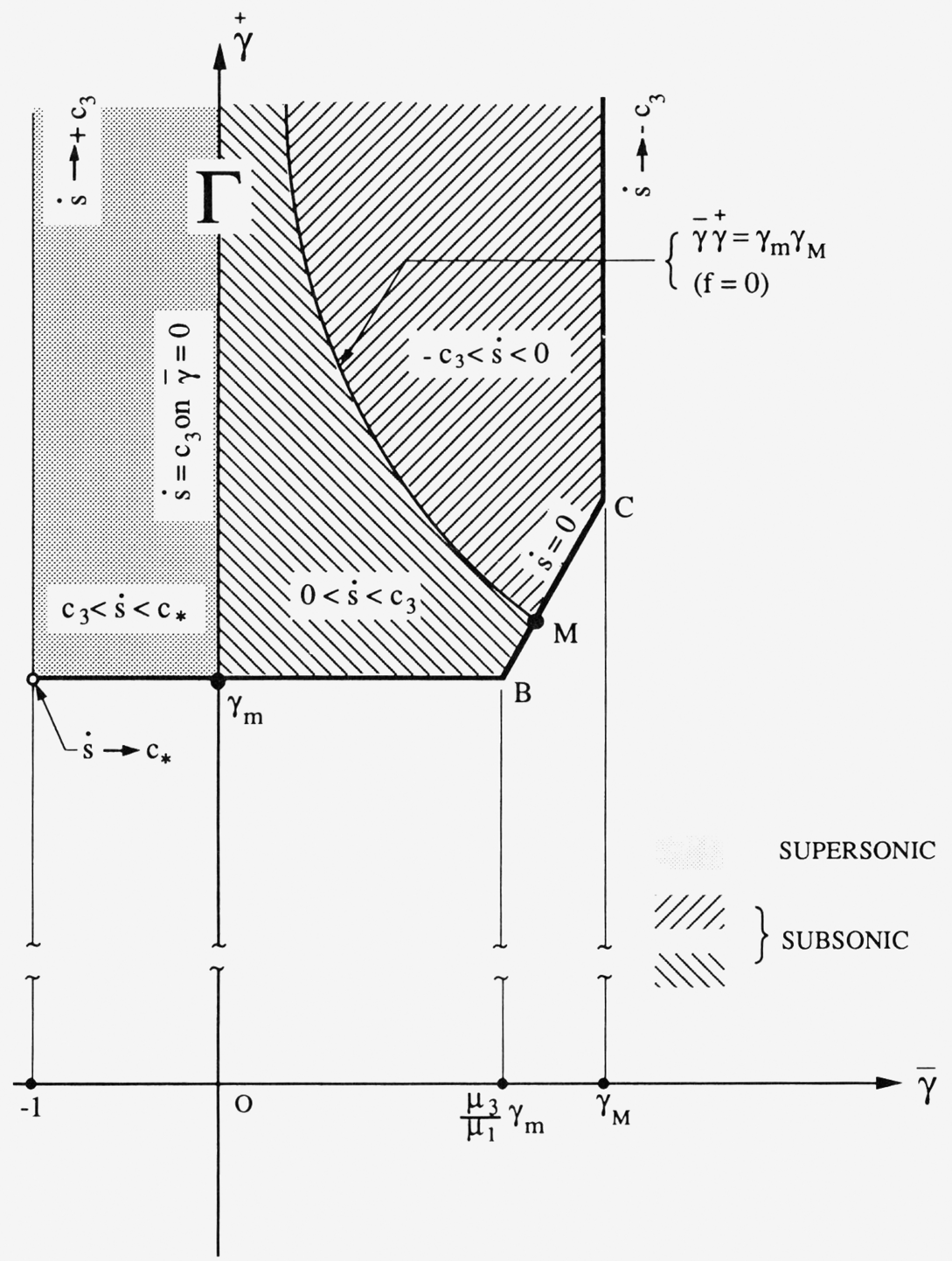

Fig. 2. The region $\Gamma$ in the $(\bar{\gamma}, \stackrel{+}{\gamma})$-plane.

propagates without dissipation. Note that the Maxwell point $M$ lies on this hyperbola. Given any point $(\bar{\gamma}, \stackrel{+}{\gamma}) \in \Gamma$, equation (3.4) determines the corresponding value of $\dot{s}^{2}$. If $(\bar{\gamma}, \hat{\gamma})$ lies off the hyperbola, then $f \neq 0$, so that the entropy admissibility requirement (2.13) determines the sign of $\dot{s}$ (see Fig. 2). For points on the hyperbola, one has $f=0$, so that the sign of $\dot{s}$ is not determined by (2.13), and propagation in either direction is possible.

With the help of (3.4) and (2.13), one can show (as in [1]) that the velocity $\dot{s}$ of a 1,3-phase boundary necessarily lies in the range

$$
-c_{3}<\dot{s}<c_{*}
$$


where

$$
c_{*}=\left(\frac{c_{1}^{2}+\gamma_{m} c_{3}^{2}}{1+\gamma_{m}}\right)^{1 / 2}, \quad c_{3}<c_{*}<c_{1} .
$$

It is helpful to note that $\rho c_{*}^{2}$ is the slope of the chord joining the point $(-1, \hat{\sigma}(-1))$ to $\left(\gamma_{m}, \sigma_{m}\right)$ in the $(\gamma, \sigma)$-plane (Fig. 1). The propagation speed $|\dot{s}|$ is said to be subsonic if $|\dot{s}|<c_{3}$. By (3.4), this occurs only for those points in the region $\Gamma$ of Fig. 2 for which $\bar{\gamma}>0$. For supersonic motion of the phase boundary, one has $\bar{\gamma}<0$, so that part of the bar is in compression.

We now use (3.4) and (3.5) to map the region $\Gamma$ of the $(\bar{\gamma}, \stackrel{+}{\gamma})$-plane into the $(\dot{s}, f)$-plane. Each point $(\bar{\gamma}, \stackrel{+}{\gamma})$ that does not lie on $B C$ (Fig. 2$)$ is carried to two points $(\dot{s}, f)$ and $(-\dot{s}, f)$ in the $(\dot{s}, f)$-plane; if $f \neq 0$, only one of these satisfies the entropy admissibility requirement $(2.13)$. If $f=0$, the point $(\bar{\gamma}, \stackrel{+}{\gamma})$ lies on the hyperbola; such a point maps to a pair of admissible points $( \pm \dot{s}, 0)$ in the $(\dot{s}, f)$ plane, with $0 \leq \dot{s} \leq c_{3}$. All points on $B C$ map to admissible points on the $f$-axis. All points in $\Gamma$ that lie on $\bar{\gamma}=0$ map to the same pair of points $\left( \pm c_{3}, f_{0}\right)$, where the constant $f_{0}$ is given by

$$
f_{0}=\frac{1}{2}\left(\mu_{1}-\mu_{3}\right) \gamma_{m} \gamma_{M}>0 \text {. }
$$

Figure 3 shows the admissible image of $\Gamma$ in the $(\dot{s}, f)$-plane. It will be useful for later purposes to note from (3.4), (3.5), and Fig. 2 that the boundary curves $B^{\prime} R^{\prime}$ and $C^{\prime} V^{\prime}$ in Fig. 3 are characterized by

$$
\begin{array}{lr}
f=f_{M}(\dot{s}) \equiv f_{0}(1-1 / \beta(\dot{s})), & 0 \leq \dot{s}<c_{3}, \\
f=f_{m}(\dot{s}) \equiv f_{0}(1-\beta(\dot{s})), & -c_{3}<\dot{s} \leq 0,
\end{array}
$$

respectively, where

$$
\beta(\dot{s})=\frac{c_{1}^{2}-\dot{s}^{2}}{c_{3}^{2}-\dot{s}^{2}} \frac{\gamma_{M}}{\gamma_{m}}, \quad-c_{3}<\dot{s}<c_{3} .
$$

4. The maximally dissipative kinetic relation. As was shown in [1] and will be reviewed briefly in Sec. 5 of the present paper, the Riemann problem for the trilinear material associated with the field equations (2.3), (2.4), the jump conditions (2.5), (2.6), and the entropy inequality (2.13) suffers from a massive failure of uniqueness of solution. We view this nonuniqueness as resulting from a constitutive deficiency in the theory reflecting the need for additional information characterizing the initiation and evolution of phase boundaries. The physical basis of phase transitions in solids, as described in the materials science literature, involves both a nucleation criterion governing the initiation of the transition and a kinetic relation controlling the rate at which it proceeds (see, for example, [5, 23]). Simple continuum-mechanical implementations of these notions were discussed in $[1,6,7]$. In particular, it was shown in [1] that, as long as all phase boundaries propagate subsonically, a nucleation criterion and a kinetic relation can be imposed in Riemann problems, and together they single out unique solutions that fulfill the entropy admissibility requirement. 


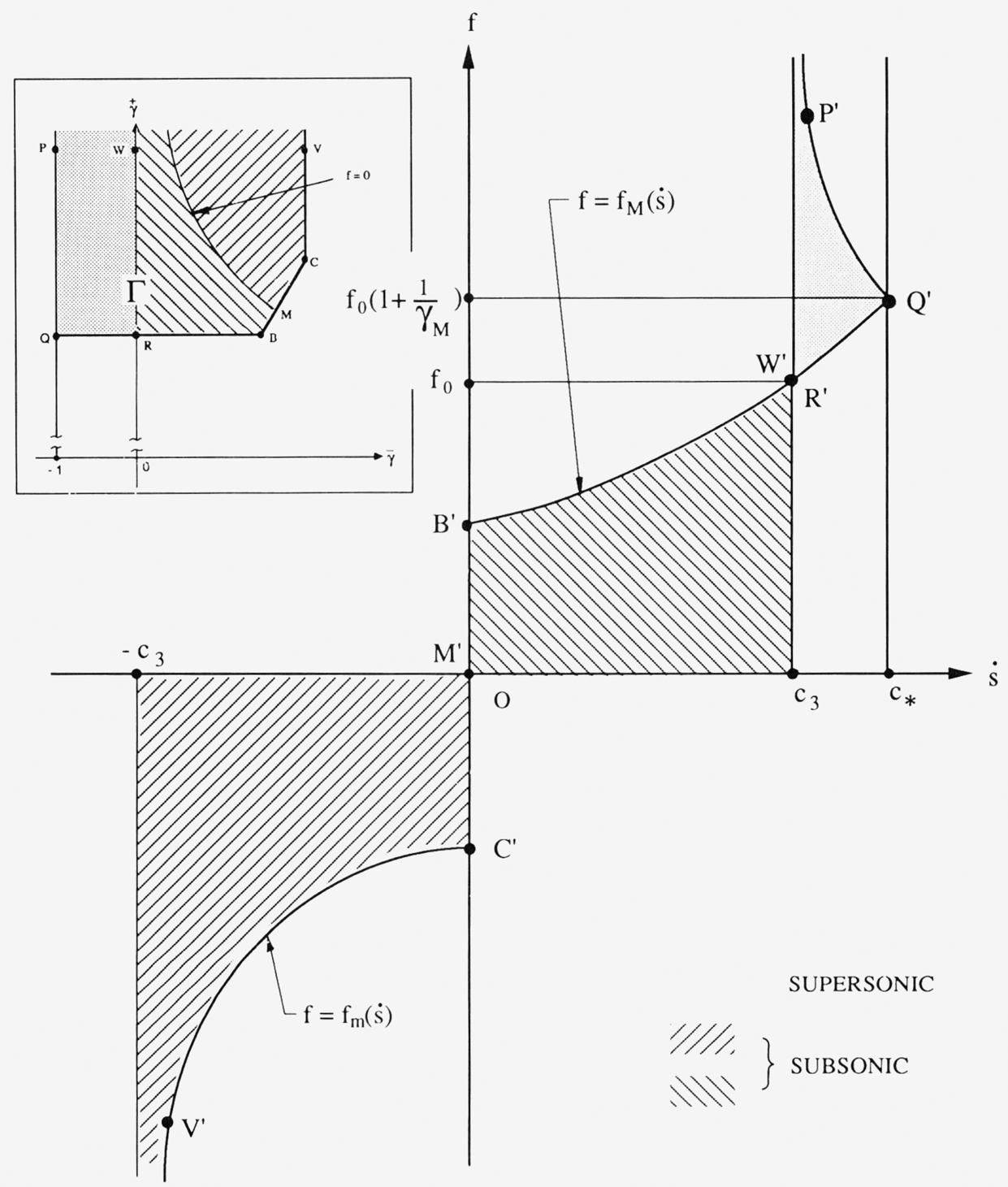

FIG. 3. Admissible image of $\Gamma$ (inset) in $(\dot{s}, f)$-plane.

Our attention in the present study will be focused on two particular kinetic relations; we shall not address the issue of nucleation here. We proceed as in [1] and assume that, if the phase boundary velocity $\dot{s}$ is subsonic, there is a function $\varphi$ determined by the material that relates the driving traction $f$ to the propagation speed $\dot{s}$, the latter being a measure of the rate at which the phase transition takes place. Thus we take

$$
f=\varphi(\dot{s}), \quad-c_{3}<\dot{s}<c_{3} .
$$

Alternatively, if $\varphi$ is monotonic on $\left(-c_{3}, c_{3}\right)$, one can write this in the inverse form,

$$
\dot{s}=V(f), \quad-\infty<f<f_{0},
$$


where the constant $f_{0}$ is given by (3.8) and the function $V$ is the inverse of $\varphi$. Because of the entropy admissibility requirement (2.13), $\varphi$ and $V$ must satisfy

$$
\varphi(\dot{s}) \dot{s} \geq 0 \quad \text { for }-c_{3}<\dot{s}<c_{3} ; \quad V(f) f \geq 0 \quad \text { for }-\infty<f<f_{0} .
$$

The curve in the $(\dot{s}, f)$-plane described by $(4.1)$ or $(4.2)$ is required to lie in the hatched region shown in Fig. 3.

Since the driving traction $f(t)$ is determined through $(2.11)$ by the strains $\stackrel{ \pm}{\gamma}(t)$ at the particles on either side of the phase boundary, the statement of the kinetic relation (4.1) involves only the quantities $\frac{ \pm}{\gamma}(t)$ and $\dot{s}(t)$ at the phase boundary and is thus purely local in character. Statement (4.1) is also problem-independent. Moreover, a relation of the form (4.1) can be generalized to three-dimensional thermomechanical processes in thermoelastic materials, as shown in [7].

The basic principles of continuum theory do not impose any further restrictions on, or provide examples of, particular kinetic functions $\varphi$ or $V$. These must be supplied by appropriate constitutive modeling. For example, the kinetic response function given by $V(f)=k \sinh (f / K)$, where $K$ and $k$ are constants, can be motivated by arguments based on thermal activation theory (the latter theory is discussed, for example, in Chapter 1 of [23]). The choice $\varphi(\dot{s}) \equiv 0$ for the kinetic response function would result if one were to require all motions of the bar to be dissipation-free. At the other extreme, there is a kinetic response function that corresponds to a definite notion of maximum dissipation, as we shall now show.

To this end, we first extend the definitions (3.9), (3.10) of $f_{M}(\dot{s})$ and $f_{m}(\dot{s})$ to the interval $\left(-c_{3}, c_{3}\right)$ by setting $f_{M}(\dot{s})=0$ for $-c_{3}<\dot{s}<0$ and $f_{m}(\dot{s})=0$ for $0<\dot{s}<c_{3}$. In terms of these extensions $f_{M}$ and $f_{m}$, the upper and lower boundaries of the hatched regions in the $(\dot{s}, f)$-plane of Fig. 3 are $f=f_{M}(\dot{s})$ and $f=f_{m}(\dot{s})$, $-c_{3}<\dot{s}<c_{3}$. We shall say that a subsonically moving phase boundary that separates phase 1 on the left from phase 3 on the right is maximally dissipative if at each instant $t$

$$
f(t) \dot{s}(t) \geq f_{*} \dot{s}(t) \quad \text { for all } f_{*} \text { such that } \quad f_{m}(\dot{s}(t)) \leq f_{*} \leq f_{M}(\dot{s}(t)) .
$$

Thus the local dissipation rate at a maximally dissipative phase boundary is at least as great as the dissipation rate during any "virtual motion" of that discontinuity whose speed $\dot{s}(t)$ at time $t$ coincides with that of the actual motion, but for which the instantaneous virtual driving traction $f_{*}$ may take on any value in the range allowed by the given value of $\dot{s}(t)$. This notion of maximum dissipation is closely related to the concept of maximum dissipation-or maximum plastic work-utilized in the constitutive description of rate-independent elastic-plastic solids [8,9].

It follows from (4.4) that if a phase boundary is maximally dissipative, then the driving traction $f(t)$ at time $t$ must coincide with $f_{m}(\dot{s}(t))$ if $-c_{3}<\dot{s}(t)<0$ and with $f_{M}(\dot{s}(t))$ if $0<\dot{s}(t)<c_{3}$. If $\dot{s}(t)=0$, (4.4) imposes no restriction on the current driving traction, so that $f(t)$ may take any value in the interval $\left[f_{m}(0), f_{M}(0)\right]$ that corresponds to the range of possible values of driving traction $f$ 


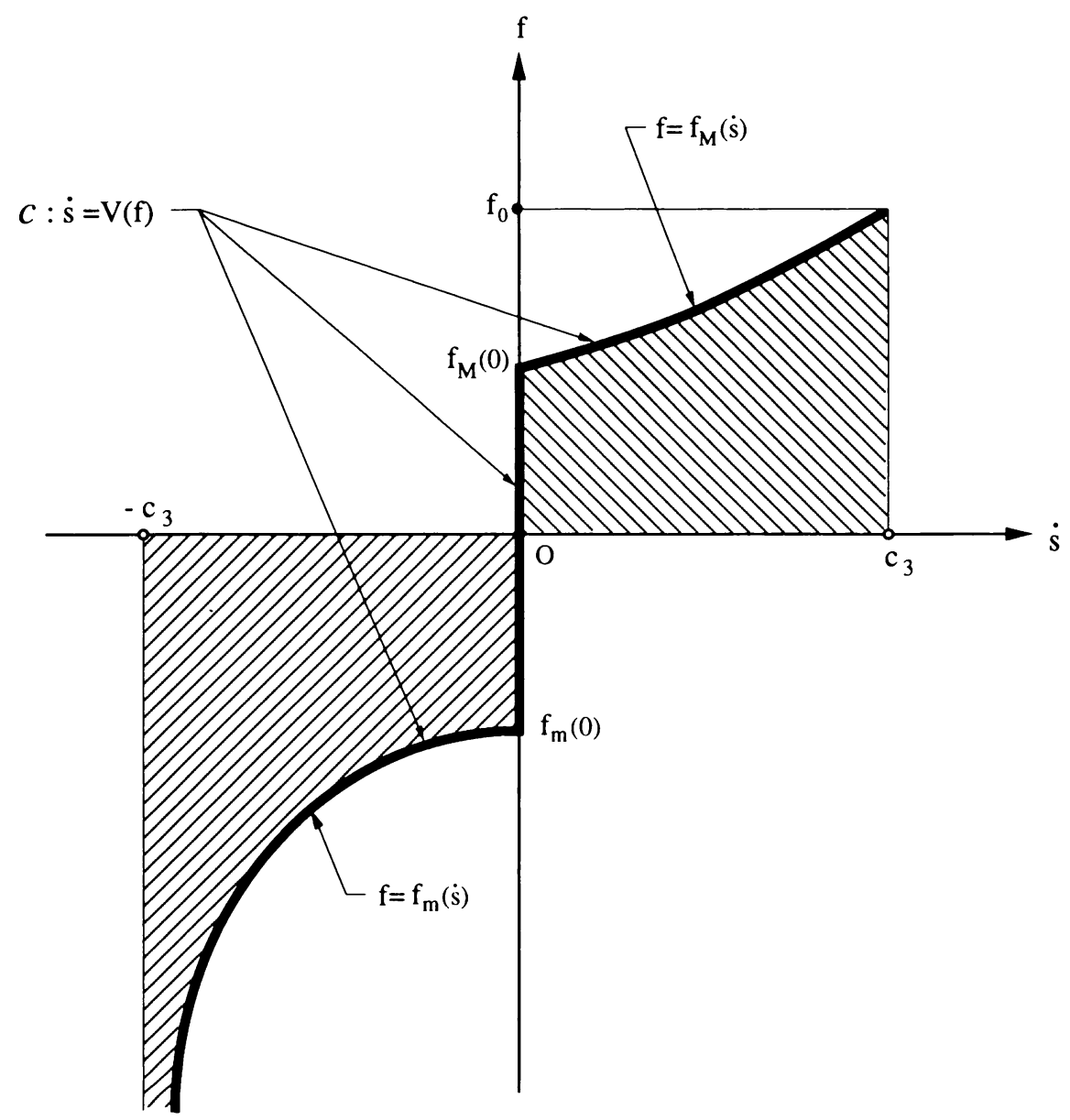

FIG. 4. Maximally dissipative kinetic relation.

for equilibrium phase boundaries. Summarizing, (4.4) holds if and only if

$$
\left.\begin{array}{ll}
f(t)=f_{m}(\dot{s}(t)) & \text { if }-c_{3}<\dot{s}(t)<0, \\
f_{m}(0) \leq f(t) \leq f_{M}(0) & \text { if } \dot{s}(t)=0, \\
f(t)=f_{M}(\dot{s}(t)) & \text { if } 0<\dot{s}(t)<c_{3} .
\end{array}\right\}
$$

In the $(\dot{s}, f)$-plane, the points $(\dot{s}(t), f(t))$ permitted by the maximum dissipation rate postulate (4.4) thus lie on the curve $C$ shown in Fig. 4 . Conversely, every point on this curve corresponds to a pair $(\dot{s}(t), f(t))$ permitted by (4.4). The curve $C$, which coincides with the boundary curve $V^{\prime} C^{\prime} T^{\prime} B^{\prime} R^{\prime}$ in Fig. 3, is reminiscent in some respects of rigid-plastic response with work-hardening or of the force-velocity relationship associated with friction. The particular kinetic relation that corresponds to $C$ is best described in the form (4.2); with the help of (3.9)-(3.11), one finds that 
the associated kinetic response function $V(f)$ is given by

$$
V(f)= \begin{cases}-c_{3}\left(\frac{f_{m}(0)-f}{\left(1-\gamma_{M} \gamma_{m}\right) f_{0}-f}\right)^{1 / 2}, & -\infty<f \leq f_{m}(0), \\ 0, & f_{m}(0) \leq f \leq f_{M}(0), \\ c_{1}\left(\frac{f-f_{M}(0)}{\left(\gamma_{m} / \gamma_{M}-1\right) f_{0}+f}\right)^{1 / 2}, & f_{M}(0) \leq f \leq f_{0},\end{cases}
$$

where $f_{0}, f_{m}(\dot{s})$, and $f_{M}(\dot{s})$ are given by (3.8)-(3.10).

Some insight into the maximally dissipative kinetic relation may be obtained by observing from (3.4), (3.5), and (3.9)-(3.11) that (4.5) implies

$$
\left.\begin{array}{ll}
\bar{\gamma}(t)=\gamma_{M} & \text { if }-c_{3}<\dot{s}(t)<0, \\
\dot{\gamma}(t)=\gamma_{m} & \text { if } 0<\dot{s}(t)<c_{3} .
\end{array}\right\}
$$

When $\dot{s}>0$, the 1,3-phase boundary advances into material that is in phase 3 and so particles of the bar are transforming from phase 3 (the "parent phase") to phase 1 (the "product phase"); thus, according to $(4.7)_{2}$, parent phase particles which are on the verge of undergoing this transformation have a strain $\stackrel{+}{\gamma}(t)=\gamma_{m}$. Similarly, (4.7) $)_{1}$ shows that during the reverse transformation from phase 1 to phase 3 , particles in the parent phase that are about to transform have a strain $\bar{\gamma}(t)=\gamma_{M}$. Thus, in terms of the stress response described in Fig. 1, a particle can cross over from the first branch of the stress-strain curve to the third branch only at the local maximum $\gamma=\gamma_{M}$, while the reverse transition can only occur at the local minimum $\gamma_{m}$.

5. A Riemann problem. We now formulate a Riemann problem for the field equations and jump conditions (2.3)-(2.6) and determine all piecewise smooth solutions to it that are invariant under a scale change $x \rightarrow k x, t \rightarrow k t$. The entropy admissibility condition is imposed ab initio, but we postpone enforcement of the maximally dissipative kinetic relation until the next section. We thus seek all scale-invariant weak solutions of the differential equations (2.3), (2.4) on the upper half of the $(x, t)$-plane that satisfy the entropy admissibility requirement and the following initial conditions:

$$
\gamma(x, 0+)=\left\{\begin{array}{ll}
\gamma_{L}, & -\infty<x<0, \\
\gamma_{R}, & 0<x<+\infty,
\end{array} \quad v(x, 0+)= \begin{cases}v_{L}, & -\infty<x<0, \\
v_{R}, & 0<x<+\infty .\end{cases}\right.
$$

Here $\gamma_{L}, \gamma_{R}, v_{L}$, and $v_{R}$ are given constants with $\gamma_{L}$ in phase 1 and $\gamma_{R}$ in phase 3:

$$
\gamma_{L} \in\left(-1, \gamma_{M}\right], \quad \gamma_{R} \in\left[\gamma_{m}, \infty\right)
$$

we say this Riemann problem is of 1,3-type. For the trilinear material, a detailed analysis of Riemann problems of this type as well as of types 1,1 and 3,3 may be found in [1]. Here we simply summarize without proof those results from [1] that are relevant for cur present purposes.

As argued in [1], solutions of the Riemann problem that are scale-invariant must have the form:

$$
\gamma(x, t)=\gamma_{j}, \quad v(x, t)=v_{j}, \quad \dot{s}_{j} t \leq x \leq \dot{s}_{j+1} t, j=0,1, \ldots, N,
$$




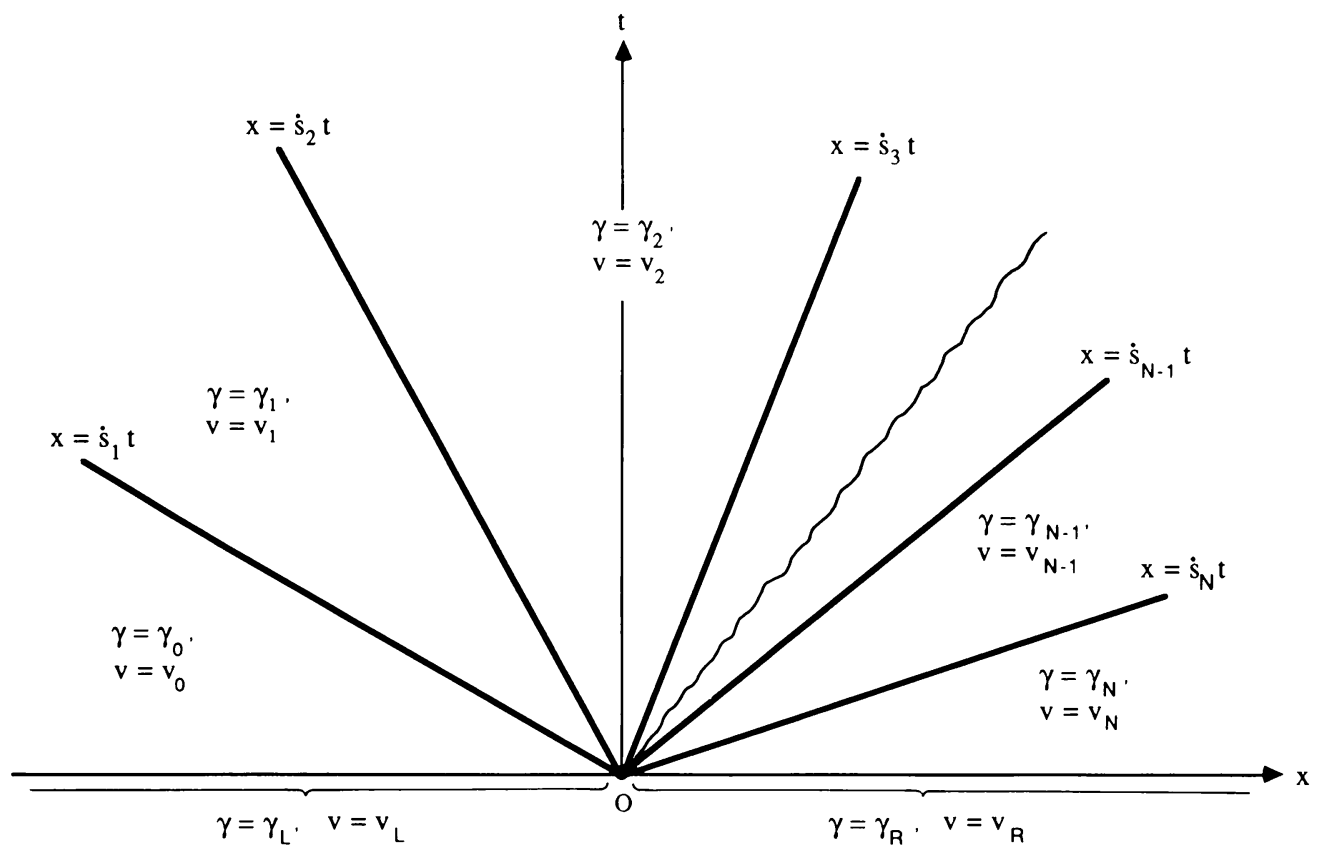

FIG. 5. Assumed form of solutions to the Riemann problem.

where $\gamma_{j}, v_{j}, \dot{s}_{j}$, and $N$ are constants, with $N$ a positive integer, and $\gamma_{0}=$ $\gamma_{L}, \gamma_{N}=\gamma_{R}, v_{0}=v_{L}, v_{N}=v_{R}, \dot{s}_{0}=-\infty$, and $\dot{s}_{N+1}=+\infty$ (see Fig. 5). The $\gamma_{j}$ 's are required to satisfy $\gamma_{j}>-1$ for $j=0, \ldots, N$ and $\gamma_{j} \neq \gamma_{j+1}$ for $j=0,1, \ldots, N-1$. In the space-time field given by $(5.3)$, there are $N$ strain discontinuities on lines $x=\dot{s}_{j} t$; they may be shock waves or phase boundaries. For the trilinear material, fans cannot occur. If $x=\dot{s}_{j} t$ is a shock wave, then $\dot{s}_{j}$ must take one of the four values $\pm c_{1}, \pm c_{3}$. We seek solutions of the Riemann problem in the class of all functions of the form described above.

At each discontinuity, the jump conditions (2.5), (2.6) must be satisfied, so that

$$
\begin{aligned}
\dot{s}_{j}\left(\gamma_{j}-\gamma_{j-1}\right) & =-\left(v_{j}-v_{j-1}\right), \\
\hat{\sigma}\left(\gamma_{j}\right)-\hat{\sigma}\left(\gamma_{j-1}\right) & =-\rho \dot{s}_{j}\left(v_{j}-v_{j-1}\right), \quad j=1, \ldots, N, \quad
\end{aligned}
$$

where $\hat{\sigma}$ is the stress response function for the trilinear material (Fig. 1). If $f_{j}$ denotes the driving traction on the discontinuity at $x=\dot{s}_{j} t$, the entropy admissibility condition (2.13) requires that

$$
f_{j} \dot{s}_{j} \geq 0, \quad j=1, \ldots, N .
$$

An admissible solution of the Riemann problem is a pair $\gamma(x, t), v(x, t)$ of the form (5.3) such that (5.4), (5.5) hold.

Let $(\gamma, v)$ be an admissible solution of the 1,3-Riemann problem described above. Then the following are immediate consequences of the results proved in [1]:

(i) no strain $\gamma_{j}$ in (5.3) belongs to phase 2 ;

(ii) $(\gamma, v)$ involves precisely one phase boundary; 


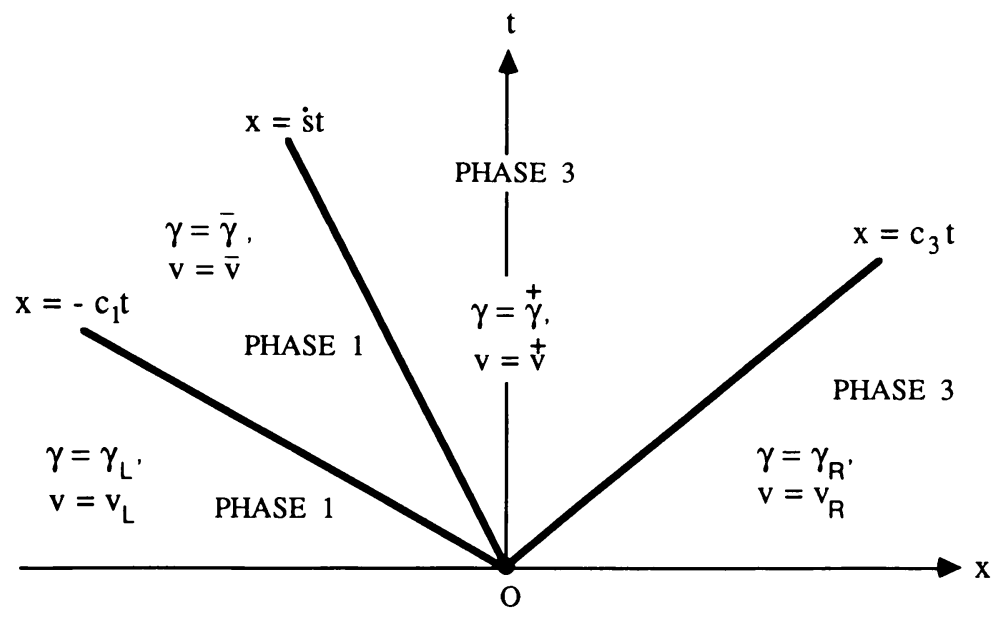

(a) SUBSONIC PHASE BOUNDARY

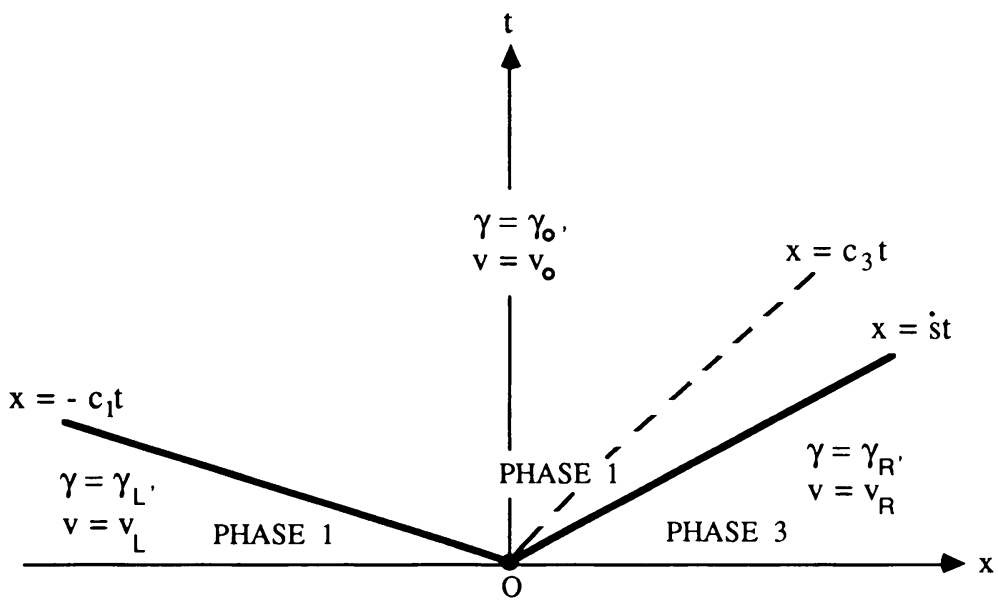

(b) SUPERSONIC PHASE BOUNDARY

FIG. 6. Form of admissible solutions to Riemann problem with 1,3initial data.

(iii) if the phase boundary travels at subsonic speed, then $N=3$ and the solution involves two shock waves as well; in this case $\dot{s}_{1}=-c_{1}, \dot{s}_{2}=\dot{s}, \dot{s}_{3}=+c_{3}$, with $-c_{3}<\dot{s}<c_{3}$ (see Fig. 6(a));

(iv) if the phase boundary travels at a supersonic speed, then $N=2$ and the solution involves one shock wave as well; in this case $\dot{s}_{1}=-c_{1}$ and $\dot{s}_{2}=\dot{s}>$ $c_{3}$ (see Fig. 6(b)).

Thus the structure of the solution to the present Riemann problem necessarily has one of the two forms shown in Fig. 6.

For the supersonic case of Fig. $6 \mathrm{~b}$, we have shown in [1] that no kinetic relation can 
be prescribed at the phase boundary. Since our present purpose is to introduce and discuss various kinetic relations, we restrict attention from here on to the subsonic case of Fig. 6a. In conformity with the result in (iii) above, we thus take $N=3$ and seek $\gamma, v$ in the form:

$$
\gamma, v= \begin{cases}\gamma_{L}, v_{L}, & -\infty<x<-c_{1} t, \\ \bar{\gamma}, \bar{v}, & -c_{1} t<x<\dot{s} t, \\ \dot{\gamma},+ & \dot{s}, \quad x<c_{3} t, \\ \gamma_{R}, v_{R}, & c_{3} t<x<\infty,\end{cases}
$$

where $\bar{\gamma}, \bar{v}, \stackrel{+}{\gamma}, \stackrel{+}{v}$, and $\dot{s}$ are unknowns, with

$$
0<\bar{\gamma} \leq \gamma_{M}, \quad \dot{\gamma} \geq \gamma_{m}, \quad-c_{3}<\dot{s}<c_{3} .
$$

The jump conditions (5.4) lead to four equations involving these five unknowns, and from them, one can determine $\stackrel{+}{\gamma}, \stackrel{+}{v}, \bar{\gamma}$, and $\bar{v}$ in terms of $\dot{s}$ :

$$
\begin{aligned}
\bar{\gamma}=\frac{c_{3}+\dot{s}}{c_{1}+\dot{s}} h, & \stackrel{+}{\gamma}=\frac{c_{1}-\dot{s}}{c_{3}-\dot{s}} h, \\
\bar{v}=v_{L}-c_{1} \gamma_{L}+\frac{c_{3}+\dot{s}}{c_{1}+\dot{s}} c_{1} h, & \stackrel{+}{v}=v_{R}+c_{3} \gamma_{R}-\frac{c_{1}-\dot{s}}{c_{3}-\dot{s}} c_{3} h,
\end{aligned}
$$

where we have set

$$
h=\left(c_{1} \gamma_{L}+c_{3} \gamma_{R}+v_{R}-v_{L}\right) /\left(c_{1}+c_{3}\right) .
$$

By using (5.8), the restrictions (5.7) can be reduced to

$$
\gamma_{m} \frac{c_{3}-\dot{s}}{c_{1}-\dot{s}} \leq h \leq \gamma_{M} \frac{c_{1}+\dot{s}}{c_{3}+\dot{s}}, \quad-c_{3}<\dot{s}<c_{3} .
$$

Note that (5.10) shows that $h$ is necessarily positive in the present subsonic case. In the $(\dot{s}, h)$-plane of Fig. 7, the inequalities $(5.10)$ describe the region on and between the top and bottom curves $C_{1}$ and $C_{2}$, whose respective equations are $h=\gamma_{M}\left(c_{1}+\dot{s}\right) /$ $\left(c_{3}+\dot{s}\right)$ and $h=\gamma_{m}\left(c_{3}-\dot{s}\right) /\left(c_{1}-\dot{s}\right),-c_{3}<\dot{s}<c_{3}$. The special ordinates $h_{m}, h_{0}$, and $h_{M}$ appearing in Fig. 7 are given by

$$
h_{m}=\sigma_{m}\left(\mu_{1} \mu_{3}\right)^{-1 / 2}, \quad h_{0}=\sigma_{0}\left(\mu_{1} \mu_{3}\right)^{-1 / 2}, \quad h_{M}=\sigma_{M}\left(\mu_{1} \mu_{3}\right)^{-1 / 2} \text {. }
$$

Let initial data be given such that $h$ in (5.9) is positive. For each set of such initial data, Fig. 7 shows that there is a range of $\dot{s}$ in which (5.10) holds. Keeping the initial data-and therefore $h$-fixed, define $\bar{\gamma}, \stackrel{+}{\gamma}, \bar{v}$, and $\stackrel{+}{v}$ by $(5.8), \gamma(x, t)$ and $v(x, t)$ by (5.6), for each $\dot{s}$ in the allowable range. For the given initial data, the set of all such pairs $\gamma, v$ so constructed comprises a one-parameter family (parameter $\dot{s}$ ) of solutions to the corresponding Riemann problem, in each of which there is a phase boundary moving at subsonic velocity $\dot{s}$. Given the initial data and a value of $\dot{s}$ in the appropriate range, there is exactly one solution to the Riemann problem and one corresponding point $(\dot{s}, h)$ on or between the curves $C_{1}$ and $C_{2}$ in the $(\dot{s}, h)$-plane.

We now determine which among the one-parameter family of solutions corresponding to given initial data with $h>0$ are admissible according to the entropy 


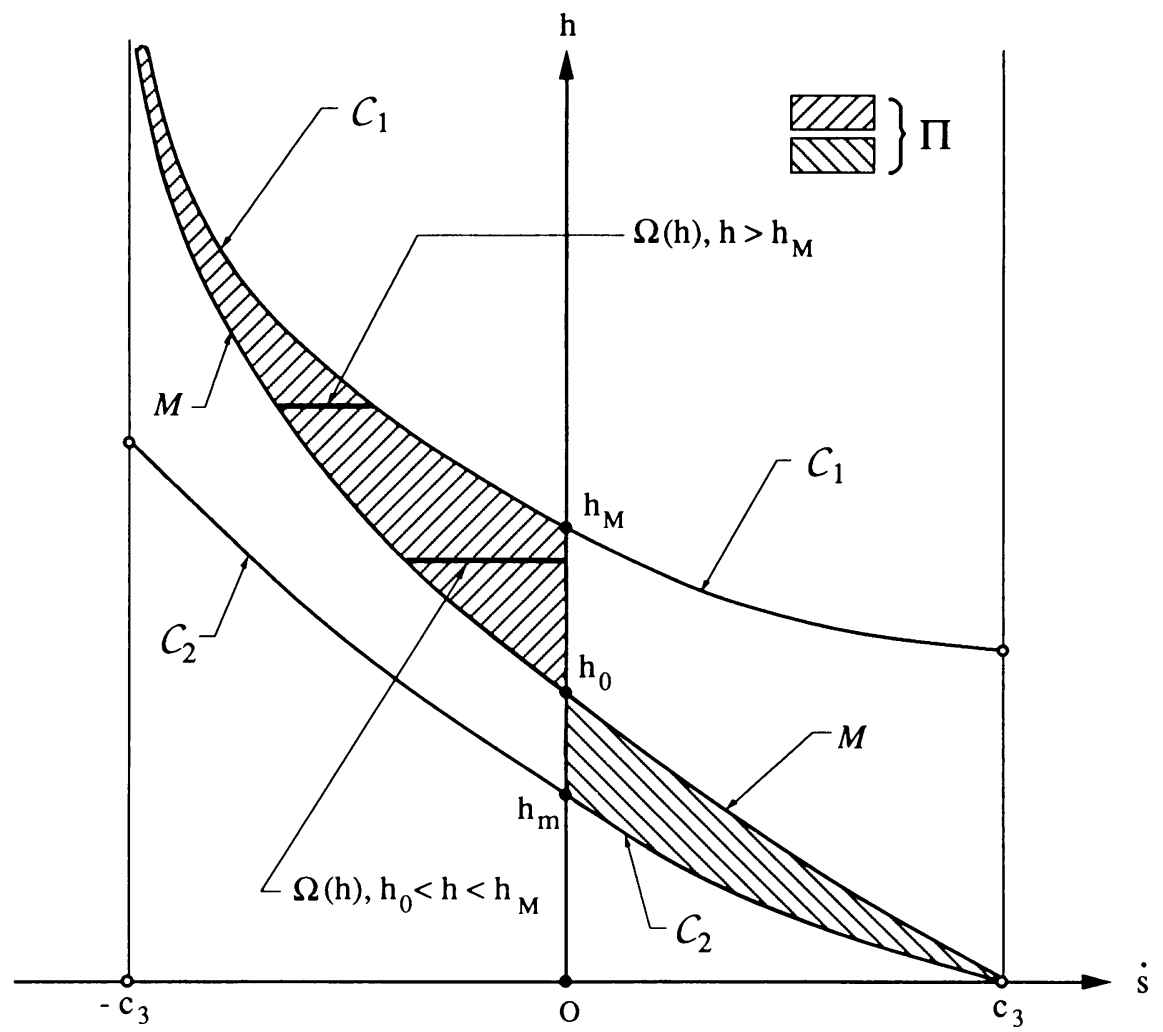

FIG. 7. The $(\dot{s}, h)$-plane.

requirement (5.5). Recall first from Sec. 3 that the driving traction on each of the two shock waves is zero, so that they automatically satisfy the entropy admissibility requirement. On the other hand, the driving traction $f$ on the phase boundary is given by (3.5) with $\bar{\gamma}, \stackrel{+}{\gamma}$ given by $(5.8)_{1,2}$ :

$$
f=\frac{1}{2}\left(\mu_{1}-\mu_{3}\right)\left\{\gamma_{m} \gamma_{M}-\frac{\left(c_{3}+\dot{s}\right)\left(c_{1}-\dot{s}\right)}{\left(c_{3}-\dot{s}\right)\left(c_{1}+\dot{s}\right)} h^{2}\right\} .
$$

With this $f$, the entropy admissibility condition (5.5) reduces to

$$
\left\{\gamma_{m} \gamma_{M}-\frac{\left(c_{3}+\dot{s}\right)\left(c_{1}-\dot{s}\right)}{\left(c_{3}-\dot{s}\right)\left(c_{1}+\dot{s}\right)} h^{2}\right\} \dot{s} \geq 0 .
$$

Let $\Pi$ stand for the set of all pairs $(\dot{s}, h)$ that are consistent with both inequalities (5.10) and (5.13); $\Pi$ thus corresponds to the union of the two hatched regions in Fig. 7. The curve $M$ that forms part of the boundary of $\Pi$ is the locus of points $(\dot{s}, h)$ for which the contents of the braces in (5.13) vanish, and thus on which $f=0$. For each $h>0$, let $\Omega(h)$ be the corresponding "horizontal" cross-section of $\Pi$ (Fig. 7):

$$
\Omega(h)=\{(\dot{s}, h) \mid h>0 \text { fixed, }(\dot{s}, h) \in \Pi\} .
$$

Let initial data be given and fixed, and suppose the associated $h$ is positive; points in the set $\Omega(h)$ then correspond one-to-one to entropically admissible solutions of the 
associated 1,3-Riemann problem, so that such points may be used to label the oneparameter family of admissible solutions corresponding to the given data. Note, that for every $h>0$, one end-point of $\Omega(h)$ lies on the "Maxwell curve" $M$; thus for each set of initial data leading to a positive $h$, there is one solution of the associated Riemann problem with $f=0$ at the phase boundary, which therefore propagates without dissipation.

Since for each $h \neq h_{0}$ the set $\Omega(h)$ is a line segment, it is clear that-as expectedthe entropy admissibility requirement does not deliver uniqueness of solution of the Riemann problem. Because $\Omega\left(h_{0}\right)$ contains only a single point, those special initial data that give rise to $h=h_{0}$ lead to a Riemann problem that has a unique admissible solution. This solution has a stationary phase boundary $(\dot{s}=0)$ bearing zero driving traction, and it tends for large time to an equilibrium mixture of phases 1 and 3 in which the bar is at the Maxwell stress $\sigma_{0}$. From Fig. 7 it is clear that $\Omega(h)$ includes points with $\dot{s}=0$ for all values of $h$ in the interval $\left[h_{m}, h_{M}\right]$. For initial data whose $h$ is in this interval but differs from $h_{0}$, the solution with $\dot{s}=0$ gives rise to a long-time mixed-phase equilibrium that is not a Maxwell state $(f \neq 0)$ and hence is metastable.

We showed in [1] that, for given initial data with $h>0$, a kinetic relation $f=\varphi(\dot{s})$ will pick out a unique admissible solution to the Riemann problem from among those corresponding to points in $\Omega(h)$, provided $\varphi$ satisfies certain conditions. We turn now to the implications for the 1,3-Riemann problem of the maximally dissipative kinetic relation constructed in Sec. 4.

6. The maximally dissipative kinetic relation and the Riemann problem. As shown in the preceding section, for initial data with $h>0$, the 1,3-Riemann problem has a one-parameter family of admissible solutions (5.6), (5.8), each with a subsonically moving phase boundary. We now seek to determine which among this continuum of solutions conforms to the maximally dissipative kinetic relation (4.2), (4.6).

Substituting (5.12) into (4.6) and then putting the result into (4.2) allows one to solve for $\dot{s}$ in terms of $h$ :

$$
\dot{s}= \begin{cases}c_{1}\left(\frac{h_{m}-h}{\gamma_{m}-h}\right) & \text { for } 0<h \leq h_{m}, \\ 0 & \text { for } h_{m} \leq h \leq h_{M}, \\ -c_{3}\left(\frac{h-h_{M}}{h-\gamma_{M}}\right) & \text { for } h_{M} \leq h<\infty .\end{cases}
$$

Let initial data with positive $h$ be given; (6.1) then determines a unique value of $\dot{s}$ such that $(\dot{s}, h) \in \Omega(h)$; once $\dot{s}$ is known, (5.8) yield $\bar{\gamma}, \bar{v}, \stackrel{+}{\gamma}, \stackrel{+}{v}$, so that $\gamma(x, t)$ and $v(x, t)$ are fully determined through (5.3). The result is the unique solution of the 1,3-Riemann problem that is consistent with the maximally dissipative kinetic relation, and of course with the entropy admissibility requirement as well. Solutions picked out by this kinetic relation for various sets of initial data can be described graphically in the $(\dot{s}, h)$-plane; they correspond to points on the curve $K$, shown bold in Fig. 8, whose equation is $(6.1)$ and which is the image in the $(\dot{s}, h)$-plane of the kinetic curve $C$ in the $(\dot{s}, f)$-plane (Fig. 4). Note that this curve coincides 


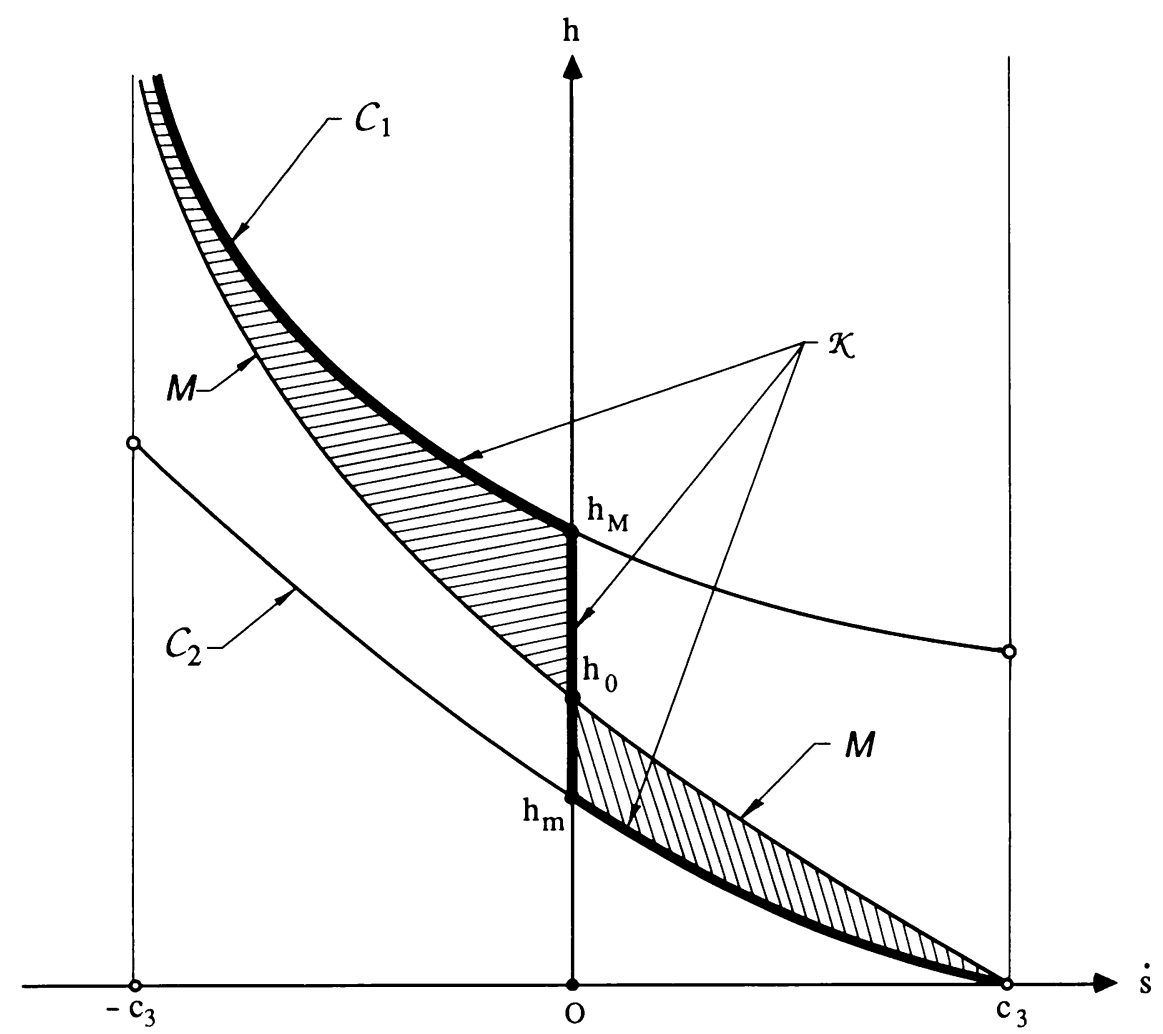

Fig. 8. The curve $K$ in the $(\dot{s}, h)$-plane corresponding to solutions of the Riemann problem selected by the maximally dissipative kinetic relation.

with a portion of the boundary of the hatched set $\Pi$. One sees from the graph that, for initial data whose associated $h$ lies in the interval $\left[h_{m}, h_{M}\right]$, the maximally dissipative kinetic relation always picks the solution with stationary phase boundary; for this solution, the long-time limit is an equilibrium mixture of phases 1 and 3 . If $0<h<h_{m}$, the kinetic relation picks a solution with $\dot{s}>0$, so that all particles of the bar are ultimately in phase 1 ; if $h>h_{M}$, the entire bar is ultimately in phase 3 . For values of $h$ in $\left(0, h_{0}\right)$, this kinetic relation picks the solution that corresponds to the left boundary point of $\Omega(h)$, while for $h>h_{0}$, it picks the right boundary point.

7. Solutions of the Riemann problem with largest dissipation rate. We now return to the Riemann problem and its admissible subsonic solutions as constructed in Sec. 5 prior to the imposition of the maximally dissipative kinetic relation. For fixed initial data with a positive value of $h$, we seek from among the one-parameter family of solutions corresponding to $\Omega(h)$ that solution whose dissipation rate is greatest.

For each solution in this family, dissipation arises only from the moving phase boundary. Thus the dissipation rate at time $t$ associated with any piece $\left[x_{1}, x_{2}\right]$ of the bar that includes $x=\dot{s} t$ in its interior is found from (2.10) and (5.12) to be 
given by

$$
D=d(\dot{s} ; h) \equiv \frac{1}{2}\left(\mu_{1}-\mu_{3}\right)\left\{\gamma_{m} \gamma_{M}-\frac{\left(c_{3}+\dot{s}\right)\left(c_{1}-\dot{s}\right)}{\left(c_{3}-\dot{s}\right)\left(c_{1}+\dot{s}\right)} h^{2}\right\} \dot{s}, \quad(\dot{s}, h) \in \Pi ;
$$

$D$ depends on $\dot{s}$ and the initial data, but is independent of $t$. We speak of $d(\dot{s}, h)$ as the dissipation rate associated with the solution of the Riemann problem that corresponds to a specified $\dot{s}$ and given data. By $(5.13), d(\dot{s} ; h) \geq 0$ for all $(\dot{s}, h) \in \Pi$. For every $h>0$, let $\dot{s}_{\max }(h)$ be such that

$$
d\left(\dot{s}_{\max }(h) ; h\right)=\max _{(\dot{s}, h) \in \Omega(h)} d(\dot{s} ; h) .
$$

It can be shown that, for each $h>0$, there is exactly one $\dot{s}_{\max }(h)$. Choosing $\dot{s}=$ $\dot{s}_{\max }(h)$ picks out the admissible solution that dissipates most rapidly in comparison with all other admissible solutions that are available for the given initial data.

Let $J$ be the curve in the $(\dot{s}, h)$-plane that corresponds to such solutions with greatest dissipation rate for various sets of initial data:

$$
J=\left\{(\dot{s}, h) \mid \dot{s}=\dot{s}_{\max }(h), h>0\right\} .
$$

We now show that, at least at some of its points, the curve $J$ does not coincide with the curve $K$ (Fig. 8) determined by the maximally dissipative kinetic relation of Sec. 4. For any $h \neq h_{0}$ in the interval, $\left[h_{m}, h_{M}\right]$, one has $\dot{s}=0$ at one end-point of the segment $\Omega(h)$ (Fig. 7), so from (7.1), $d=0$ there. Similarly, the other end-point of $\Omega(h)$ lies on the Maxwell curve $M$ where $f=0$, so $d$ vanishes there as well. At interior points of $\Omega(h), d$ is positive. It follows that, for $h_{m}<h<h_{M}, h \neq h_{0}$, the maximizing point $\left(\dot{s}_{\max }(h), h\right)$ for $d(\dot{s} ; h)$ lies in the interior of the line segment $\Omega(h)$ and therefore in particular not on the $h$-axis. In contrast, for this same range of values of $h$, all points $(\dot{s}, h)$ on the curve $K$ lie on the $h$-axis. Thus the curves $K$ and $J$ do not coincide everywhere.

More detailed information concerning the curve $J$ can be found by investigating the locus in the $(\dot{s}, h)$-plane of horizontal tangents of $d(\dot{s} ; h)$ as a function of $\dot{s}$ for fixed $h$. To this end, define

$$
\Delta(\dot{s}) \equiv\left(c_{1}^{2}-\dot{s}^{2}\right)\left(c_{3}^{2}-\dot{s}^{2}\right)+2\left(c_{1}-c_{3}\right) \dot{s}\left(c_{1} c_{3}+\dot{s}^{2}\right), \quad-c_{3} \leq \dot{s} \leq c_{3} .
$$

It is easy to verify that $\Delta\left(-c_{3}\right)<0, \Delta(\dot{s})>0$ for $0 \leq \dot{s} \leq c_{3}$, and that $\Delta(\dot{s})$ increases monotonically with $\dot{s}$ on the interval $\left[-c_{3}, 0\right]$. Thus there is a unique number $\dot{s}_{*}$ in $\left(-c_{3}, 0\right)$ such that $\Delta\left(\dot{s}_{*}\right)=0, \Delta(\dot{s})<0$ for $-c_{3} \leq \dot{s}<\dot{s}_{*}$, and $\Delta(\dot{s})>0$ for $\dot{s}_{*}<\dot{s}<c_{3}$. Direct calculation shows that, if $(\dot{s}, h)$ is a point that lies on the locus of horizontal tangents of $d$, then necessarily $\Delta(\dot{s})>0$, so that $\dot{s}_{*}<\dot{s}<c_{3}$; moreover, on this locus, $h$ and $\dot{s}$ are related by

$$
h=H(\dot{s}) \equiv h_{0} \frac{\left(c_{1}+\dot{s}\right)\left(c_{3}-\dot{s}\right)}{\{\Delta(\dot{s})\}^{1 / 2}}, \quad \dot{s}_{*}<\dot{s}<c_{3} .
$$

It can be shown that $H(\dot{s})$ decreases as $\dot{s}$ increases on $\left(\dot{s}_{*}, c_{3}\right)$, and that only part of the curve $J_{*}$ represented by (7.5) lies in $\Pi$. The locus $J$ of maxima of $d(\dot{s} ; h)$ thus consists of the union of that portion of $J_{*}$ that lies in $\Pi$ together with appropriate 


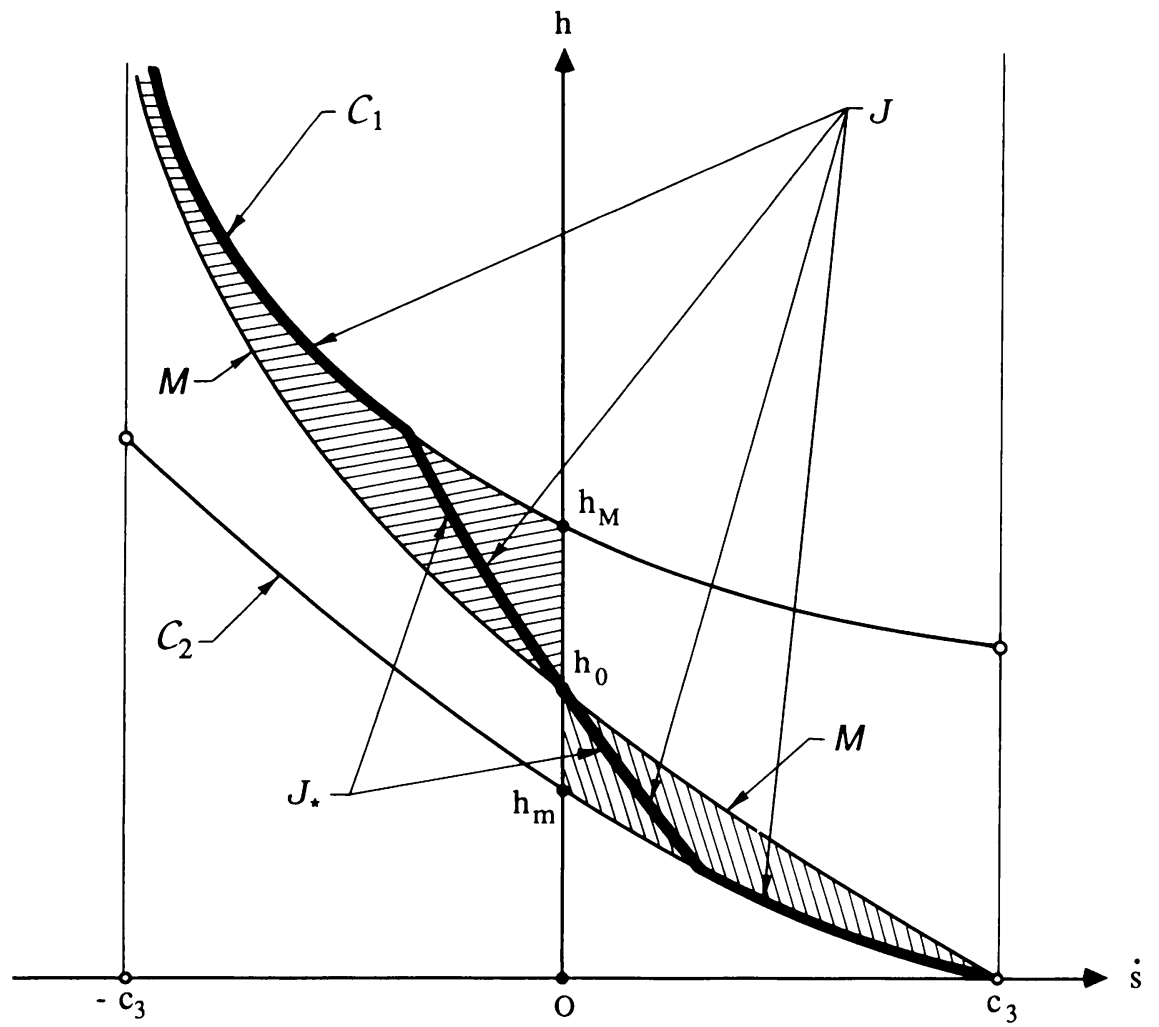

FIG. 9. The curve $J$ in the $(\dot{s}, h)$-plane associated with the modified entropy rate shock admissibility criterion.

parts of the boundary curves $C_{1}$ and $C_{2}$, so that the curves $J$ and $K d o$ coincide in part. Figure 9 shows a representative instance of the curve $J$ as computed numerically for a particular set of values of the material parameters.

Since $h$ varies monotonically with $\dot{s}$ on $J$, selecting the solution to a given 1,3-Riemann problem that maximizes $d(\dot{s} ; h)$ over $\Omega(h)$ for the appropriate $h$ represents a criterion that does indeed single out a unique admissible solution for each set of initial data with positive $h$. Since the curves $J$ (Fig. 9) and $K$ (Fig. 8 ) are not the same, this selection criterion differs from the one furnished by the maximally dissipative kinetic relation of Sec. 4. Indeed, in the latter case, (4.4) shows that maximization of the dissipation rate $f \dot{s}$ is carried out for fixed $\dot{s}$, i.e., on vertical cross-sections of $\Pi$, while in the criterion leading to $J$, maximization takes place for fixed $h$, and therefore on horizontal cross-sections of $\Pi$.

The selection criterion described in the present section appears to be closely related to the "entropy rate shock admissibility criterion" as stated by Dafermos on p. 56 of [17]. In the context of the isothermal trilinear elastic bar and in our terminology, a motion would be considered admissible by this criterion if each of its propagating strain discontinuities fulfills the following condition at each time instant $t_{*}$ : let $\stackrel{+}{\gamma}\left(t_{*}\right), \stackrel{+}{v}\left(t_{*}\right), \bar{\gamma}\left(t_{*}\right)$, and $\bar{v}\left(t_{*}\right)$ be the instantaneous strains and particle velocities at a discontinuity, and let $D_{*}$ be the associated instantaneous local dissipation 


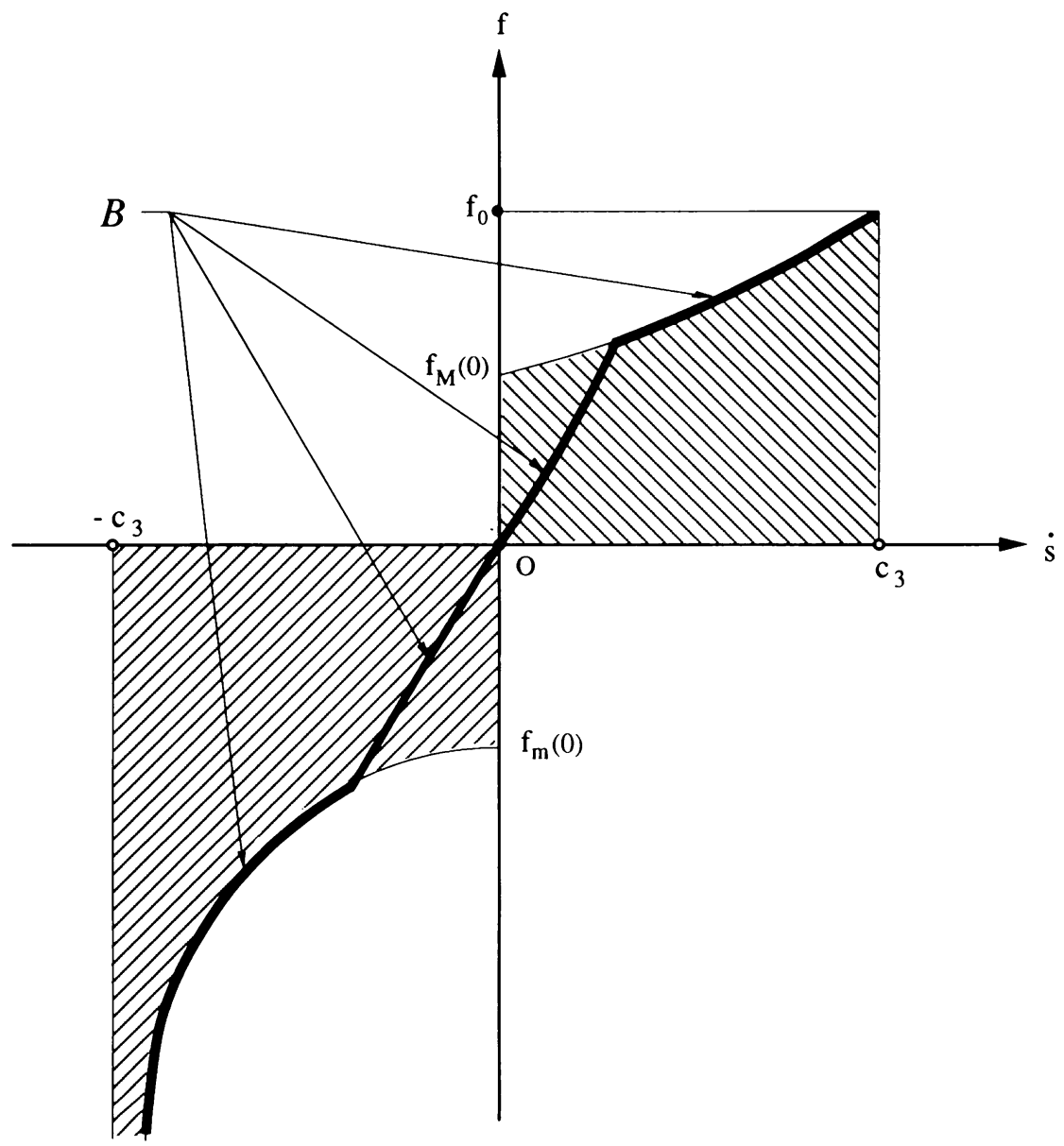

FIG. 10. Kinetic relation $B$ corresponding to the modified entropy rate shock admissibility criterion.

rate; let $\tau=t-t_{*}$ be a new time; then no solution of the Riemann problem in the $(x, \tau)$-plane with $\stackrel{+}{\gamma}\left(t_{*}\right), \stackrel{+}{v}\left(t_{*}\right), \bar{\gamma}\left(t_{*}\right)$, and $\bar{v}\left(t_{*}\right)$ as initial data has a dissipation rate greater than $D_{*}$. As we understand this criterion, it does not impose what we have called the entropy admissibility requirement (2.13) on the solutions of the Riemann problem that compete in the process of maximizing the dissipation rate. If this requirement were imposed, then the maximization process associated with the resulting modified version of Dafermos's criterion, when applied to a discontinuity of 1,3-type, would seem to coincide with the "horizontal" maximization scheme described in detail above. Moreover, the selection process associated with this "modified entropy rate shock admissibility criterion" can be characterized by a kinetic relation of the form $f=\varphi(\dot{s})$; the appropriate $\varphi$ can be found by mapping the curve $J$ from the $(\dot{s}, h)$-plane to the $(\dot{s}, f)$-plane with the help of $(5.12)$ and (7.5). For a particular set of material parameters, the corresponding kinetic response curve $B$ in the $(\dot{s}, f)$-plane is shown in Fig. 10 . 
The distinction between the sets of competing solutions to the Riemann problem that enter the modified and unmodified version of the entropy rate shock admissibility criterion is illustrated by the counterpart in the present context of a result of Hattori [18]. For the van der Waals fluid, Hattori shows that the Maxwell equilibrium mixture of what we would call phases 1 and 3 is admissible according to the entropy rate criterion of Dafermos. In our setting, reference to Fig. 7 shows that a horizontal line through the "Maxwell point" $\dot{s}=0, h=h_{0}$ would intersect the set $\Pi$ of entropically admissible points in the single point $\left(0, h_{0}\right)$. In contrast, the intersection of this same line with the set of points corresponding to all solutions to the Riemann problem of the form (5.6) is the horizontal line segment through $\left(0, h_{0}\right)$ connecting $C_{2}$ to $C_{1}$. Each solution of the form (5.6) that corresponds to a point other than $\left(0, h_{0}\right)$ on this line segment involves a negative dissipation rate and is thus inadmissible by the entropy requirement (2.13). Thus the set of competitors appropriate to the modified version of the entropy rate criterion contains only the Maxwell equilibrium solution. In contrast, among the continuum of competitors entering the unmodifed version of the criterion, all except the Maxwell equilibrium solution are inadmissible according to the entropy inequality (2.13). Since the dissipation rate associated with the Maxwell equilibrium phase mixture is zero, this state can represent that solution of the Riemann problem whose dissipation rate is greatest only if all competitors are inadmissible according to the second law of thermodynamics as manifested by (2.13). The special initial data for which $h=h_{0}$ correspond to the exceptional circumstance in which the entropy admissibility requirement alone is sufficient for uniqueness of scale-invariant solutions to the Riemann problem for the trilinear material.

More generally, if the entropy inequality is not imposed upon the competitors entering Dafermos's shock admissibility criterion, then in the present setting, the required maximization must be carried out over all solutions of the form (5.3) that satisfy the given initial conditions, and not merely over the set of solutions of the simpler form (5.6) involving only a single phase boundary. The entropy admissibility requirement plays a major role in the arguments used in [1] to establish results (i)-(iv) stated in Sec. 5. It is these results in turn that make it possible to restrict attention to solutions of the form (5.6) in the 1,3-Riemann problem for the trilinear material.

Combining the conclusions reached in the present paper with those established in [22], we may assert that, at least for the trilinear material, a modified version of the entropy rate shock admissibility criterion of [17], a criterion of the viscositycapillarity type [10-15], and a maximally dissipative kinetic relation motivated by the notion of maximum plastic work can all be subsumed under the class of phase transitions whose kinetics are characterized by relations of the form $f=\varphi(\dot{s})$ between driving traction and phase boundary velocity.

Acknowledgment. The support of the Mechanics Branch of the U.S. Office of Naval Research through Contract N00014-87-K-0117 and Grant N00014-90-J-1871 is gratefully acknowledged. 


\section{REFERENCES}

[1] R. Abeyaratne and J. K. Knowles, Kinetic relations and the propagation of phase boundaries in solids, Arch. Rational Mech. Anal. 114, 119-154 (1991)

[2] O. A. Oleinik, On the uniqueness of the generalized solution of the Cauchy problem for a nonlinear system of equations occurring in mechanics, Uspekhi Mat. Nauk (N.S.) 12, no. 6 (78), 169-176 (1957) (Russian)

[3] T. P. Liu, Uniqueness of weak solutions of the Cauchy Problem for general $2 \times 2$ conservation laws, J. Differential Equations 20, 369-388 (1976)

[4] C. M. Dafermos, Discontinuous thermokinetic processes, Appendix 4B of Rational Thermodynamics (C. Truesdell, ed.), Springer-Verlag, New York, 1984

[5] J. W. Christian, The Theory of Transformations in Metals and Alloys, Part I, Pergamon Press, Oxford, 1975

[6] R. Abeyaratne and J. K. Knowles, On the dissipative response due to discontinuous strains in bars of unstable elastic material, Internat. J. Solids and Structures 24, 1021-1044 (1988)

[7] R. Abeyaratne and J. K. Knowles, On the driving traction acting on a surface of strain discontinuity in a continuum, J. Mech. Phys. Solids 38, 345-360 (1990)

[8] J. R. Rice, On the structure of stress-strain relations for time-dependent plastic deformation in metals, J. Appl. Mech. 37, 728-737 (1970)

[9] J. Lubliner, A maximum dissipation principle in generalized plasticity, Acta Mech. 52, 225-237 (1984)

[10] M. Shearer, Nonuniqueness of admissible solutions of Riemann initial value problems for a system of conservation laws of mixed type, Arch. Rational Mech. Anal. 93, 45-69 (1986)

[11] M. Shearer, Dynamic phase transitions in a van der Waals gas, Quart. Appl. Math. 46, 631-636 (1988)

[12] M. Slemrod, Admissibility criteria for propagating phase boundaries in a van der Waals fluid, Arch. Rational Mech. Anal. 81, 301-315 (1983)

[13] M. Slemrod, Dynamics of first order phase transitions, Phase Transformations and Material Instabilities in Solids (M. E. Gurtin, ed.), Academic Press, New York, 1984, pp. 163-203

[14] L. Truskinovsky, Equilibrium phase interfaces, Soviet Phys. Dokl. 27, 551-553 (1982)

[15] L. Truskinovsky, Structure of an isothermal phase discontinuity, Soviet Phys. Dokl. 30, 945-948 (1985)

[16] C. M. Dafermos, The entropy rate admissibility criterion for solutions of hyperbolic conservation laws, J. Differential Equations 14, 202-212 (1973)

[17] C. M. Dafermos, Hyperbolic systems of conservation laws, Systems of Nonlinear Partial Differential Equations (J. M. Ball, ed.), Reidel, Dordrecht, 1983, pp. 25-70

[18] H. Hattori, The Riemann problem for a van der Waals fluid with entropy rate admissibility criterion. Isothermal case, Arch. Rational Mech. Anal. 92, 247-263 (1986)

[19] H. Hattori, The Riemann problem for a van der Waals fluid with entropy rate admissibility criterion. Nonisothermal case, J. Differential Equations 65, 158-174 (1986)

[20] R. D. James, The propagation of phase boundaries in elastic bars, Arch. Rational Mech. Anal. 73, 125-158 (1980)

[21] T. J. Pence, On the encounter of an accoustic shear pulse with a phase boundary in an elastic material: energy and dissipation, J. Elasticity, to appear

[22] R. Abeyaratne and J. K. Knowles, Implications of viscosity and strain gradient effects for the kinetics of propagating phase boundaries in solids, Technical Report No. 11, ONR contract N00014-87-K0117, April 1990; to appear in SIAM J. Appl. Math.

[23] D. A. Porter and K. E. Easterling, Phase Transformations in Metals and Alloys, van NostrandReinhold, New York, 1981 